\title{
Subserosal intramural ectopic pregnancy in an adenomyotic area following assisted reproduction treatment
}

\author{
Ahmed Abdel-Gadir • Kishor Shah • \\ Oluseye O. Oyawoye $\cdot$ Bina P. Chander
}

Received: 6 April 2009 /Revised: 29 April 2009 /Accepted: 30 April 2009/Published online: 30 May 2009

(C) Springer-Verlag 2009

\begin{abstract}
A 38-year-old woman presented for early pregnancy ultrasound scanning 6 weeks and 4 days following an assisted reproduction treatment cycle. She had $\beta$ human chorionic gonadotrophin ( $(\mathrm{hCG}$ ) blood level of $10,853 \mathrm{IU} / \mathrm{L} 2$ weeks before presentation. She gave previous history of termination of pregnancy, myomectomy and bilateral salpingectomy. The uterus was retroverted with multiple fibroids and non-homogenous myometrium in many areas. The endometrium was $21.1 \mathrm{~mm}$ thick with no intrauterine pregnancy. An initial diagnosis of cornual/interstitial ectopic pregnancy was made. However, 3D images rendering and the multiplanar technique showed a $27.5-\mathrm{mm}$ gestation sac, medial and above the interstitial part of the right tube, with 7.6-mm-long foetal pole. BhCG and progesterone blood levels on the same day were 19,551 IU/L and $43.2 \mathrm{nmol} / 1$, respectively. The patient opted against methotrexate treatment. An ectopic pregnancy bulging out of the fundal area was excised laparoscopically. Histopathological assessment showed chorionic villi
\end{abstract}

\footnotetext{
A. Abdel-Gadir $(\bowtie) \cdot$ B. P. Chander

London Female and Male Fertility Centre, Highgate Hospital,

17-19 View Road,

London N6 4DJ, UK

e-mail: AhmedAGadir@aol.com

K. Shah

Independent Histopathology Services,

142-144, New Cavendish Street,

London WIW 6YF, UK

O. O. Oyawoye

Department of Obstetrics and Gynaecology,

Newham University Hospital,

Glen Road, Plaistow,

London E13 8SL, UK
}

surrounded by myometrium, as well as foci of adenomyosis, reaching the outer serosa. To our knowledge, this is the second case of subserosal intramural ectopic pregnancy to be reported and the first in a subserosal area of adenomyosis.

Keywords Intramural pregnancy · Subserosal pregnancy · Subserosal adenomyosis

\section{Introduction}

Intramural pregnancies are rare and have been described as conceptions within the uterine wall, surrounded by myometrium without connection to the endometrial cavity, fallopian tubes or round ligaments $[1,2]$. A mortality rate of $2.5 \%$ has been quoted with such pregnancies [3]. Predisposing risk factors include assisted reproduction treatment (ART), uterine surgery, salpingectomy and adenomyosis.

Making the right diagnosis could be difficult with transvaginal ultrasound scanning, and cornual or interstitial pregnancies might be diagnosed instead. In most cases, the correct diagnosis is made only intraoperatively [4]. A high degree of suspicion is needed for all these varieties of ectopic pregnancies; otherwise, a diagnosis of pregnancy of unknown location would be made. One report suggested that an interstitial ectopic pregnancy should be suspected when the $\beta$ human chorionic gonadotrophin (BhCG) level $>2,000 \mathrm{IU} / \mathrm{L}$ with an empty uterus [5]. Furthermore, three criteria have been set to diagnose interstitial pregnancies: The uterine cavity should be empty, with the ectopic sac $>1 \mathrm{~cm}$ from its most lateral edge, and it should be surrounded by a thin myometrial layer [6]. Additionally, the interstitial echogenic line was found to be $80 \%$ 
sensitive and $98 \%$ specific in diagnosing interstitial pregnancies [7]. Furthermore, the myometrium surrounding the cornual type has been described as abnormally thin and less than $5 \mathrm{~mm}$ thick [8]. However, in cases of intramural pregnancies, the thickness of the myometrium around the sac depends on the degree of invasion by the ectopic trophoblasts. The single published case of subserosal intramural ectopic pregnancy [9] is a good example because of such deep implantation. Irrespective of all efforts, it might not be possible to differentiate between intramural and interstitial/cornual pregnancies.

Conservative medical treatment with systemic or local methotrexate has been used for all sorts of ectopic pregnancy including intramural ones. However, such treatment is not always successful, and surgical intervention or selective uterine arteries embolisation [10] might be necessary to deal with the persistent trophoblasts.

\section{Case report}

A 38-year-old woman with 4 years history of infertility attended our clinic for early pregnancy ultrasound scanning following an ART cycle performed overseas. Two embryos were replaced in her uterus 6 weeks and 4 days before. A BhCG blood level 2 weeks before presentation was $10,853 \mathrm{IU} / \mathrm{L}$. She gave history of previous termination of pregnancy and two myomectomies, one laparoscopic and one through open surgery, on two different occasions. A large $10-$ to $12-\mathrm{cm}$ intramural anterior fundal fibroid was removed during open surgery, and four tumours were removed during the laparoscopic procedure followed by resuturing of the uterus laparoscopically. Bilateral salpingectomy had also been performed, because of bilateral hydrosalpinges, following three unsuccessful ART attempts. She was not in pain and had no vaginal bleeding. BhCG and serum progesterone levels were 19,551 IU/L and $43.2 \mathrm{nmol} / \mathrm{l}$, respectively, on the same day.

Transvaginal scan examination showed a retroverted uterus with multiple small fibroids, the largest measuring $4.6 \times 3.8 \mathrm{~cm}$. The myometrium was non-homogenous in many areas suggestive of adenomyosis. The endometrium was $21.1 \mathrm{~mm}$ thick. There was no intrauterine pregnancy. Fibroid shadowing interfered with the quality of the pictures during 2D scanning. However, a gestational sac $27.5 \mathrm{~mm}$ in diameter was seen at the fundal area beside the largest fibroid and $6.4 \mathrm{~cm}$ deep relative to the vaginal vault (Fig. 1). An initial diagnosis of cornual/interstitial pregnancy was suspected. However, image manipulation with 3D rendering and the multiplanar technique revealed a different picture. The sac was seen bulging out of the fundal area above and medial to the echogenic interstitial part of the excised right tube (Fig. 2), a picture we have not seen

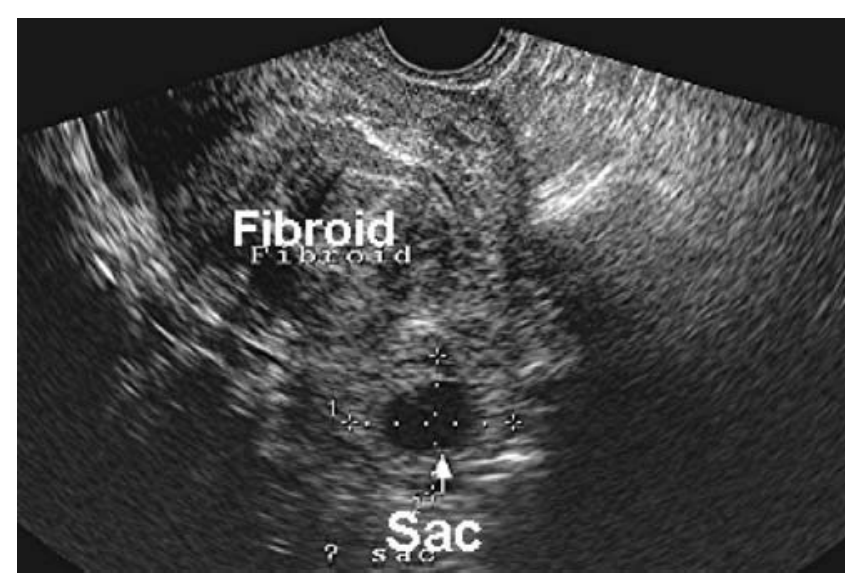

Fig. 1 An oblique transvaginal ultrasound picture showing a subserosal gestational sac with a nearby intramural fibroid. The sac was high in the fundal area and $6.4 \mathrm{~cm}$ deep relative to the vaginal vault. The body of the uterus and the empty uterine cavity are not shown in this view

before. The foetal pole was $7.6 \mathrm{~mm}$ long, equivalent to 6 weeks and 3 days pregnancy, and the yolk sac was $4.9 \mathrm{~mm}$ in diameter. There was no free fluid in the pelvis.

The diagnosis, treatment options and risks involved were discussed with the couple. The possibility of ultrasoundguided methotrexate injection [11] was excluded because of the inaccessibility of the sac. The patient was not agreeable

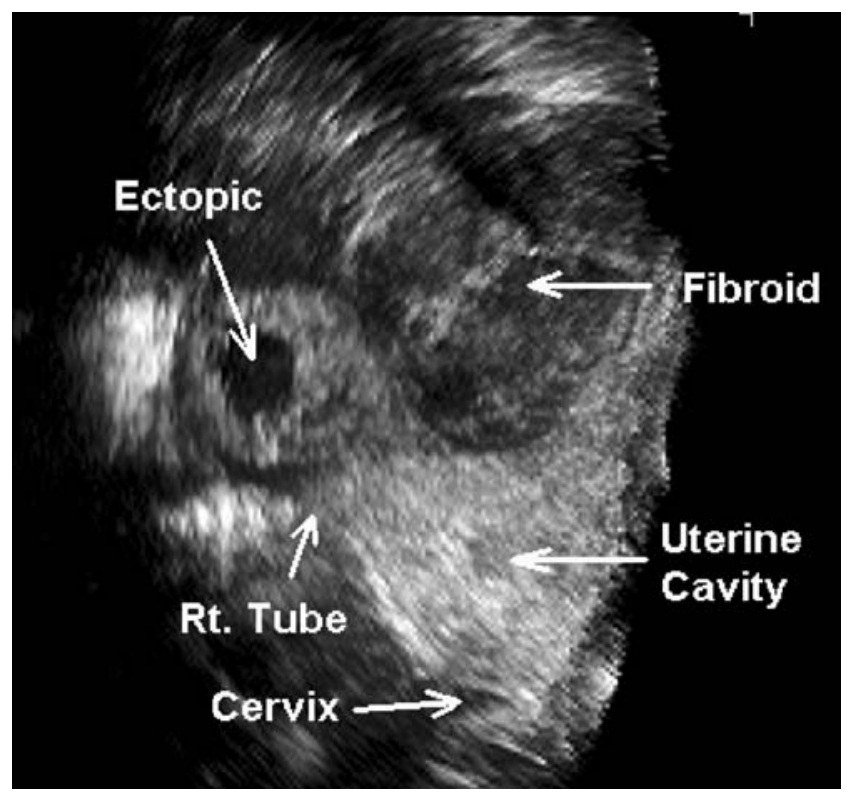

Fig. 2 A rendered coronal view of the uterus with a right fundal ectopic pregnancy above and medial to the echogenic interstitial part of the right tube with the same fibroid shown in Fig. 1 nearby. The empty triangular uterine cavity is marked with echogenic endometri$\mathrm{um}$ and is dented at the fundus by the same fibroid 
to use systemic methotrexate and opted for laparoscopic treatment instead. The risk of antenatal and intrapartum uterine rupture during any future pregnancy was thoroughly explained. She was very keen not to lose her uterus. The couple took 2 days to discuss their options and might have had a second opinion as well. They opted for laparoscopic excision of the ectopic pregnancy, with the risk of a hysterectomy explicitly explained. She consented for this option as a life-saving procedure only.

Laparoscopy revealed a large ectopic pregnancy bulging from the fundal area of the uterus with a nearby fibroid (Fig. 3). Meticulous dissection and cauterisation of all the blood vessels leading into the mass (Fig. 4) was done before it was excised down to the surface of the fundus, with minimal blood loss and minimal use of bipolar electrocautery for haemostasis, to reduce the risk of muscles necrosis (Fig. 5). Reapproximation of the edges was not done as the scar was almost level with the surface of the fundus. She was discharged from hospital on the following day. The risks involved with any further pregnancy were reemphasised to the couple during the postoperative visit.

Histological examination of the excised specimen showed trophoblastic tissue with chorionic villi surrounded by thick myometrium (Fig. 6). In addition, the muscular wall showed many foci of adenomyosis, some near the outer serosa (Fig. 7).

\section{Discussion}

This case had many of the risk factors described before, which could lead to intramural ectopic pregnancies. The

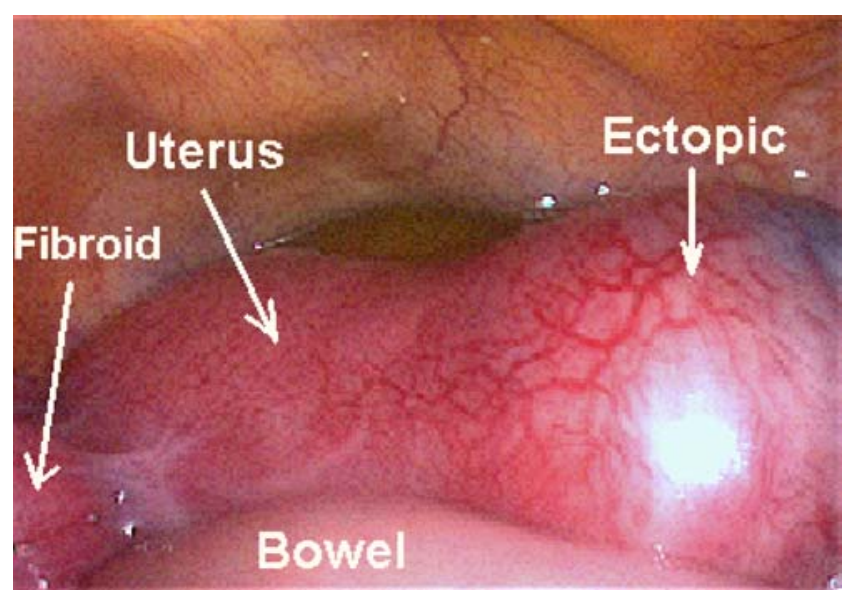

Fig. 3 A laparoscopic view showing the subserosal ectopic pregnancy protruding through the fundal area with the same fibroid shown in Figs. 1 and 2, as well as a neighbouring subserous fibroid

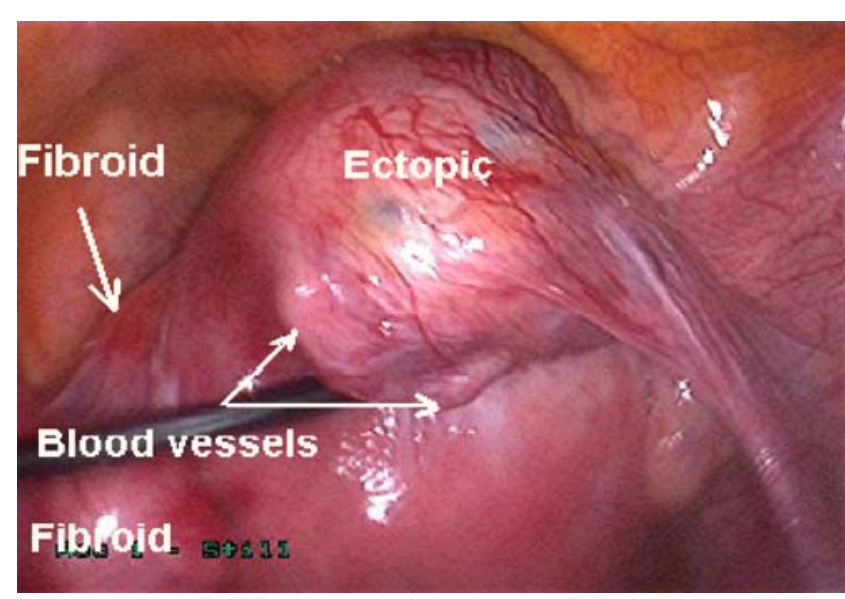

Fig. 4 A laparoscopic view following forced anteversion of the uterus with an intrauterine canulla. The ectopic mass was made more prominent by forward pressure applied on its posterior aspect with grasping forceps. Note the two prominent blood vessels marked with arrows. Other vessels were seen and cauterised on the other side and during dissection before excision of the ectopic pregnancy

huge fundal bulge of the ectopic mass, as confirmed by laparoscopy, indicated a subserosal intramural location which has been reported only once before [9]. Such deep intramuscular implantation was confirmed histopathologically and might have gone through a sinus tract created during a previous myomectomy [9]. This is especially so as the site of the ectopic pregnancy corresponded to the site of the large intramural fibroid removed by open surgery, as documented in her previous notes. The role of the concurrent adenomyosis is also important in this respect $[1,12]$ and could simulate the

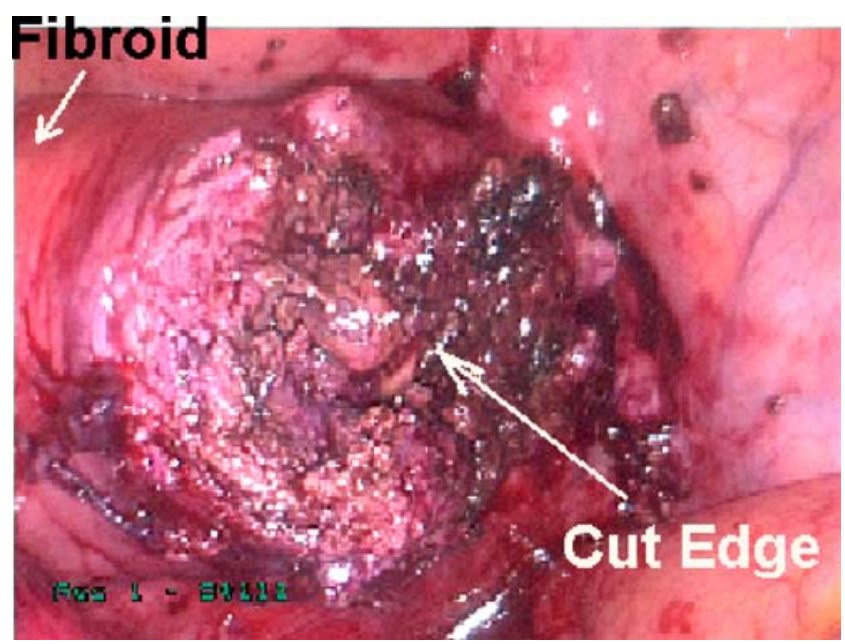

Fig. 5 A laparoscopic view of the uterus after excision of the ectopic mass down to the level of fundus 


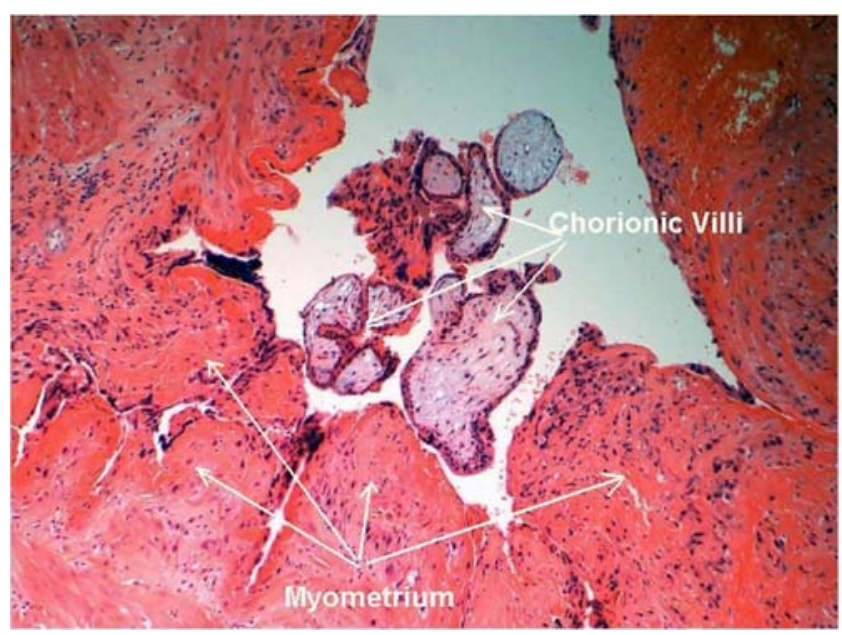

Fig. 6 A haematoxylin and eosin-stained histopathological photomicrograph $(\times 100)$ showing chorionic villi at the centre surrounded by thick myometrium with invading cytotrophoblastic cells

increased myometrial invasion by FIGO grade 1 endometrial adenocarcinoma in cases with, compared to cases without, adenomyosis [13].

Magnetic resonance imaging (MRI) has been used to help with the diagnosis of tubal and interstitial ectopic pregnancies with great success. Criteria similar to those used during ultrasound examination for the diagnosis of interstitial ectopic pregnancies $[6,7]$ have been suggested for MRI as well $[14,15]$. However, the statistics given for accuracy of transvaginal ultrasound scanning were not very much different from those quoted for MRI. The sensitivity and specificity of transvaginal ultrasonography in detecting ectopic pregnancies was $90 \%$ and $99 \%$, respectively, with positive and negative predictive values of $93.5 \%$ and $99.8 \%$, respectively. The diagnostic accuracy was $90.9 \%$ [16]. In comparison, a sensitivity of $95 \%$ and specificity of $100 \%$ in diagnosing ectopic pregnancies was quoted, respectively, for MRI with an accuracy of 96\% [17]. Accordingly, transvaginal ultrasound remains to be the first-line diagnostic imaging technique in the management of ectopic pregnancies. This is especially so because of its easy availability as an office diagnostic procedure. We see an important role for MRI in cases of pregnancies of unknown location when ultrasound fails to show intrauterine or ectopic pregnancies in patients with high positive BhCG blood levels. It could also be helpful if the uterus is distorted with multiple fibroids and a suspected diagnosis of an ectopic pregnancy is not possible to verify or exclude because of such anatomical changes and the shadowing caused by fibroids. In retrospect, we feel that MRI would not have added further useful information to that already provided by 3D scanning, in our case. The sac was seen and its exact intramural location was clearly ascertained, relative to the uterine cavity and the interstitial part of the right tube. This confirms the important role of 3D rendering in helping with the diagnosis in such difficult cases as emphasised before [2]. It definitely changed our initial impression of a cornual pregnancy, but further clinical interpretation of the intramural site as a subserosal ectopic pregnancy was missed as only one case has been published before, and we were not aware of that article at that time. The final diagnosis was made laparoscopically.

To the best of our knowledge, this is the second case of subserosal intramural ectopic pregnancy to be reported. Similar to the first case [9], there was previous history of myomectomy. However, it is the first case of subserosal intramural ectopic pregnancy in a subserosal adenomyotic area. Treatment of intramural pregnancies is difficult and no consensus has yet been agreed upon. The situation is even more difficult with the subserosal type as this is only the second case to be reported. However, like cornual pregnancies, laparoscopy could be the preferred surgical method of treatment of intramural pregnancies in experienced hands [18]. Reapproximation of the uterine scar would be necessary when the incision involves the body of the uterus itself. However, with the subserosal type, the bulging mass was excised down to the level of the fundus and reapproximation of the edges of a flat scar would be difficult or even impossible. Accordingly, minimal use of the electrocautery would be necessary to prevent muscle necrosis and further weakening of the scar. The risk of bleeding could be reduced beforehand by occluding all the blood vessels connected to the mass first, as done in this case, before excising the subserosal ectopic pregnancy itself.

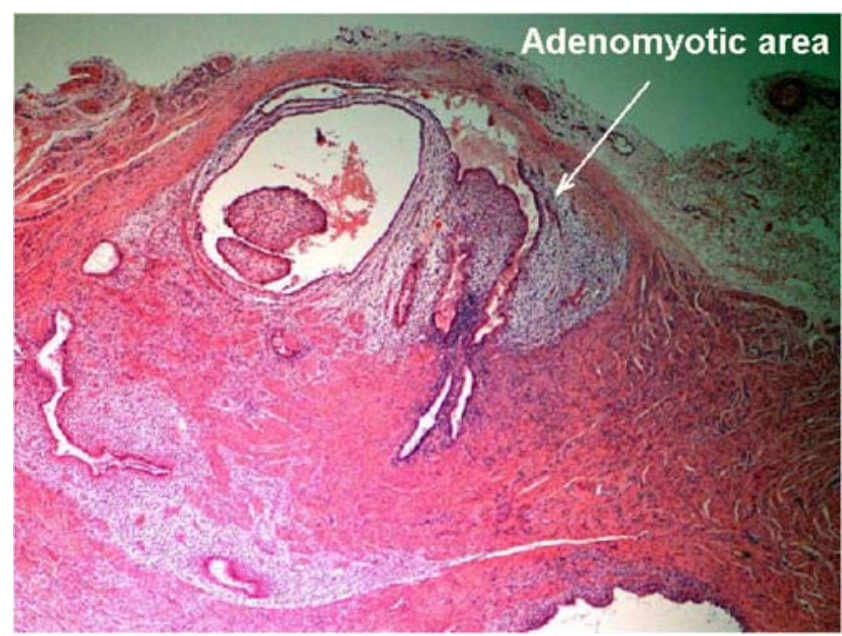

Fig. 7 A histopathological low power $(\times 40)$ haematoxylin and eosinstained section showing subserosal myometrium with a large focus of endometrial glands and stroma, typical of adenomyosis 
Conflict of interest There is no actual or potential conflict of interest in relation to this article.

\section{References}

1. Ginsburg KA, Quereshi F, Thomas M, Snowman B (1989) Intramural ectopic pregnancy in adenomyosis. Fertil Steril 51 (2):354-356

2. Caliskan E, Cakiroğlu Y, Coraqkci A (2008) Expectant management of an intramural ectopic pregnancy in a primigravid woman. Turkish-German Gynecol Assoc 9(4):234-236

3. Neiger R, Weldon K, Means N (1998) Intramural pregnancy in a caesarean section scar: a case report. J Reprod Med 43:999-1001

4. Lee GS, Hur SY, Kown I, Shin JC, Kim SP, Kim SJ (2005) Diagnosis of early intramural ectopic pregnancy. J Clin Ultrasound 33(4):190-192

5. Timmerman E, Roovers JP, Ankrum WM, Hajenius P (2008) Interstitial pregnancy: a rare type of ectopic pregnancy. Ned Tijdsch Geneesdk 152(14):787-791

6. Timor-Tritsch IE, Monteagudo A, Matera C, Weit CR (1992) Sonographic evaluation of cornual pregnancies treated with surgery. Obstet Gynecol 79:1044-1049

7. Ackerman TE, Levi CS, Dashefsky SM, Holt SC, Lindsay DJ (1993) Interstitial line: sonographic finding in interstitial (cornual pregnancy) ectopic pregnancy. Radiology 189(1):83-87

8. Fleischer AC, Manning FA, Jeanty P, Romero R (1996) Sonography in obstetrics and gynaecology. Principles and practice, 5th edn. Appleton and Lange, East Norwalk, Connecticut

9. Park WI, Jeon YM, Lee JY, Shin SY (2006) Subserosal pregnancy in a previous myomectomy site: a variant of intramural pregnancy. J Minim Invasive Gynecol 13(3):242-244
10. Yang SB, Lee SJ, Joe HS, Goo DE, Chang YW, Kim DH (2007) Selective uterine artery embolisation for management of interstitial ectopic pregnancy. Korean J Radiol 8(2):176-179

11. Oyawoye S, Chander B, Pavlovic B, Hunter J, Abdel Gadir A (2003) Heterotopic pregnancy: successful management with aspiration of cornual/interstitial gestational sac and instillation of small dose of methotrexate. Fetal Diagn Ther 18:1-4

12. Karakök M, Balat O, Sari I, Kocer NE, Erdogan R (2002) Early diagnosed intramural ectopic pregnancy associated with adenomyosis: report of an unusual case. Clin Exp Obstet Gynecol 29 (3):217-218

13. Ismiil ND, Rasty G, Ghorab Z, Nofech-Mozes S, Bernardini M, Thomas G, Ackerman I, Covens Z, Khalifa MA (2007) Adenomyosis is associated with myometrial invasion by FIGO 1 endometrial adenocarcinoma. In J Gynecol Pathol 26(3):278-283

14. Filhastre M, Dechaud H, Lesnik A, Taourel P (2005) Interstitial pregnancy: role of MRI. Eur Radiol 15(1):93-5

15. Bourdel N, Roman H, Gallot D, Lenglet Y, Dieu V, Juillard D, Linstrument C, Botchorishvili R, Rabishong B, Mage G, Canis M (2007) Interstitial pregnancy. Ultrasonographic diagnosis and contribution of MRI. A case report. Gynecol Obstet Fertil 35 (2):121-124

16. Condous G, Okaro E, Khalid A, Lu C, Van Huffel S, Timmerman D, Bourne T (2005) The accuracy of transvaginal ultrasonography for the diagnosis of ectopic pregnancy prior to surgery. Hum Reprod 20(5):1404-9

17. Yoshigi J, Yashiro N, Kinoshita T, O'uchi T, Kitagaki H (2006) Diagnosis of ectopic pregnancy with MRI: efficacy of $\mathrm{T} 2$ * $_{\text {- }}$ weighted imaging. Magn Reson Med Sci 5(1):25-32

18. Soriano D, Vicus D, Mashiach R, Schiff E, Seidman D, Goldenberg M (2008) Laparoscopic treatment of cornual pregnancy: a series of 20 consecutive cases. Fertil Steril 9 (3):839-843 